\title{
Master weaver regulates guardian
}

CCCTC-binding factor (CTCF) is a zinc finger-containing transcription factor that has many potential functions, including the regulation of higher order chromatin structure. A paper in Genes \& Development indicates that this "master weaver" of the genome is involved in the regulation of the guardian of the genome, the tumour suppressor p53, through binding to the TP53 antisense transcript WRAP53.

Previous studies have shown that WRAP53 is required for TP53 transcription in response to DNA damage. CTCF is known to bind to and induce the transcription of TP53, and the CTCF binding site in the TP53 promoter overlaps with the first intron of WRAP53 on the opposite DNA strand, suggesting that CTCF might regulate both TP53 and WRAP53. Short hairpin RNAs (shRNAs) against CTCF resulted in decreased RNA levels of both TP53 and WRAP53, and small interfering RNAs (siRNAs) against WRAP53 also reduced p53 expression levels, but the combined downregulation of WRAP53 and CTCF was not additive in terms of reducing TP53 mRNA levels, leading the authors to suggest that WRAP53 and CTCF might be physically and functionally linked. RNA immunoprecipitation and quantitative PCR indicated that CTCF and WRAP53 can interact, and this was further substantiated through the use of a genome-wide unbiased approach: that is, photoactivatable ribonucleotide-enhanced cross-linking and immunoprecipitation (PAR-CLIP). These results also showed that CTCF interacts with a wide range of RNAs. Further analyses indicated that zinc fingers 10 and 11, as well as the carboxyl terminus of CTCF, are able to interact with RNA.

What is the biological importance of these interactions? Using shRNA knockdown of endogenous CTCF in U2OS cells, the authors showed that cells exposed to a DNAdamaging agent had a defective
DNA damage response (owing to a lack of p53 expression) when a CTCF RNA-binding mutant was expressed compared with wild-type CTCF. This is presumably because the mutant CTCF cannot bind to WRAP53 RNA and so cannot induce TP53 transcription. Further experiments indicated that binding of RNAs by CTCF enables the formation of large multimers of CTCF, which might be important for chromatin looping.

These findings indicate that CTCF is able to interact with RNAs and WRAP53 in particular, and that this interaction enables the multimerization of CTCF molecules. Whether this is required for the regulation of higher order chromatin structure and how this is relevant to the regulation of $\mathrm{p} 53$ by CTCF need to be determined.

Nicola McCarthy

ORIGINAL RESEARCH PAPER Saldaña-Meyer, $R$. et al. CTCF regulates the human $p 53$ gene through direct interaction with its natural antisense transcript, Wrap53. Genes Dev. 28, 723-734 (2014) 